\title{
Editorials
}

\section{ARE WE ANY CLOSER TO BEING ABLE TO MEASURE LEPROSY EXPOSURE?}

As we reach the end of the 20th century, the goal of eliminating leprosy as a public health problem is in sight. However, case detection rates in highly endemic countries appear to remain stable. This may partially be due to changes in case ascertainment and an improved health delivery system. However, there is still no way to assess the prevalence of exposure in an endemic community. If we are to make progress towards eradication and assess the impact of MDT as it is actually delivered in situ, such a test will be needed. ${ }^{1}$ If it were possible to develop a test that would allow us to measure total exposure in a community, this could help us eventually to predict mathematically the relationship between exposure and the incidence of leprosy, and also to assess whether the risk of infection in the community is changing over time (the 'trend in the annual risk of infection') ${ }^{2}$ Such a test would need to be highly specific (i.e. non-exposed persons from non-endemic countries should be nonresponsive); there should be some way to differentiate a response due to exposure to $M$. leprae from that due to other mycobacteriae; an increase in the proportion of the population testing 'positive' for the test should predict (at least in a subset of those 'positive') an increase in the risk of acquiring disease; and finally, a 'positive' test result should be more common in sub-groups of the community known to be at higher risk of disease (i.e. household contacts, especially the very young contacts of smear-positive MB patients). ${ }^{3}$

In tuberculosis, the other mycobacterial disease that affects millions worldwide, exposure has been assessed through use of a delayed hypersensitivity (DTH) skin test reaction using a purified protein derivative (PPD). The PPD skin test's sensitivity in identifying infected persons has made it the accepted measure for assessing the incidence of infection in endemic communities, despite the fact that PPD has significant cross-reactivity with other mycobacterial antigens, especially BCG. ${ }^{4}$ There are many parallels between tuberculosis and leprosy: the number of exposed persons far exceeds those who actually develop clinical disease; there is a significant lag time between exposure and disease; both organisms engage cell-mediated immunity; and in both instances, the immune systems of a high proportion of 'exposed' individuals control the organisms soon after contact. ${ }^{5}$

The search for a skin test for leprosy is not new. Skin test reactivity to Rees' or Convit's cytosolic $M$. leprae antigen (called Leprosin A or MLSA) does not predict an increased risk for the development of clinical leprosy, rather the opposite. In longitudinal studies conducted in Malawi, any degree of skin test reactivity to soluble antigen was associated with decreased 
subsequent risk of developing leprosy. ${ }^{6}$ Further, extensive skin test surveys using MLSA in India have shown that there is no appreciable difference in reaction size distributions between leprosy patients, contacts and other persons in the endemic community. ${ }^{7,8}$ Fractionation products of $M$. leprae (MLSA produced from purified $M$. le prae from armadillo tissues minus the immunosuppressive lipid lipoarabinomannin (LAM), called MLSA-LAM, and M. leprae cell wall antigen from the same source, called MLCwA) have now been developed and produced in the USA under FDA-approved manufacturing (GMP) conditions. ${ }^{9}$ In 6-day whole blood assays, stimulation by MLSA-LAM and MLcWA led to the production of significantly more interferon-gamma (IFN) than by Rees' antigen in leprosy patients. There was also good concordance in that study between Lepromin A skin test positivity (Mitsuda reaction) and production of IFN. However, in these 6-day assays, using $\mathrm{T}$ cells from presumably non-exposed individuals from non-endemic countries, stimulation by the fractions also led to IFN production. This has been accepted as evidence that the fractions have poor specificity. ${ }^{10}$ However, the 6-day assay may not be the appropriate approach to evaluate this. A high correlation has been found between IFN production in a $24-\mathrm{h}$ whole blood assay and $48 \mathrm{~h}$ DTH reactivity to PPD in tuberculosis. ${ }^{11}$

IFN is a cytokine produced by 'armed effector' T-cells already circulating in the blood of exposed individuals and IFN produced in 24-h cultures is from these cells. ${ }^{12}$ Further, IFN is vital in the initiation of a DTH response: IFN-producing cells can be demonstrated in skin test sites (where they make up approximately $33 \%$ of cells by $48 \mathrm{~h}$ after in vivo inoculation). ${ }^{13}$ It therefore seems that the correct approach to evaluate new potential skin test antigens should use the $24 \mathrm{~h}$ assay. Twenty-four hour whole blood assays in Nepal have recently shown that the T-cells of both household contacts of leprosy patients and paucibacillary leprosy patients, when stimulated by MLSA-LAM and MLCwA, produce significantly greater amounts of IFN than the T-cells of other persons in endemic communities (socalled 'endemic controls'). T-cells of tuberculosis patients when stimulated by the same antigens do not have this response. This suggests a lesser degree of cross-reactivity with M. tuberculosis than has been found using ordinary MLSA. In the same study, on 24-h MLSA stimulation there was no difference in IFN production between 'endemic controls' and household contacts, paucibacillary leprosy patients or tuberculosis patients. ${ }^{14}$ (This MLSA was the parent substance produced in Colorado, prior to removal of LAM.) Phase I clinical safety trials in the US have further shown that these fractions are non-toxic and do not elicit a skin test reaction in individuals from non-endemic countries who have not been exposed to leprosy. ${ }^{15}$ A phase II skin test trial, which will also take place in Nepal in 2000, will investigate the appropriate dose, immunological dynamics, sensitivity and specificity of the fractions in a country with high leprosy endemicity.

From our observation of a specific IFN response to MLSA-LAM and MLCwA, we would predict that there will be larger and more frequent skin test reactions in leprosy-exposed individuals compared to 'endemic controls'. Will these antigens be 'good enough' to use in further investigations of the exposure dynamics of leprosy? The search for a highly specific leprosy skin test antigen has led the WHO TDR to fund multi-centre immunological screening of a pool of $M$. leprae-specific peptides (identified from the $M$. leprae genome sequencing project). Immunological responses to these peptide pools have been highly variable in different continents, related to the different frequency of HLA subtypes in each population. ${ }^{16} \mathrm{New}$, presumably specific peptides or even whole $M$. leprae-specific proteins will probably be identified and need to be tested in vitro in the coming few years. But even should the ideal antigen be identified, and the costly and time-consuming process of 
producing it under GMP conditions be undertaken, such a highly specific antigen is likely to be less sensitive than the current fractionation antigens (since an increase in specificity is usually accompanied by a decrease in sensitivity). ${ }^{9}$ The current fractionation products may thus be our best practical hope for a skin test, and it seems important to ensure that proper trials are done to evaluate their usefulness in countries with high leprosy endemicity, where they will need to be used eventually by control programmes. Should the current fractionation products described here prove inadequate, it will still be important to establish a reliable method to screen new potential skin test antigens: the 24-h whole blood IFN assay seems an approach worth confirmation prior to testing out an antigen in a skin test trial. The eventual development of a useful leprosy skin test will provide new opportunities for leprosy control, for rational targeting of leprosy resources and a tool to investigate the remaining epidemiological conundrums of leprosy.

\section{Reference}

1 Smith WCS. We need to know what is happening to the incidence of leprosy. Lepr Rev, 1997; 68: 195-200.

2 Sutherland I. On the risk of infection. Bull Int Union Tuberc Lung Dis, 1991; 66: 189-191.

3 Ponnighaus JM, Fine PEM, Sterne JAC, Bliss L, Wilson RJ, Malema SS, Kileta S. Incidence Rate of Leprosy in Karonga District, Northern Malawi: patterns by age, sex,. BCG status and classification. Int J Lepr, 1994; 62: $10-23$.

${ }^{4}$ Rieder HL. Methodological issues in the estimation of the tuberculosis problem from tuberculin surveys. Tubercle Lung Disease, 1995; 76: 114-121.

5 Fine P. Leprosy and tuberculosis. Tubercle, 1984; 65:137-153.

${ }^{6}$ Fine PEM, Sterne JAC, Pvnnighaus JM, Rees RJW. Delayed-type hypersensitivity, mycobacterial vaccines and protective immunity. Lancet, 1994; 344: 1245-1249.

7 Gupte MD, Anantharaman DS, Kannan S, Vallishayee RS. Experiences with Mycobacterium leprae soluble antigens in a leprosy endemic population. Lepr Rev, 1990; 61: 132-144.

8 Krishnamurthy P, Rao PS, Subramanian M, Suresh Kumar SK, Neelan PN. Mycobacterium leprae soluble antigen (Rees) skin test responses in an endemic population in India. Ind J Lepr, 1993; 65: 49-57.

9 Brennan PJ. TerLouw SM. New skin test antigens for the diagnosis of leprosy. Transactions of the 15 th International Leprosy Congress. Int J Lepr, 1998; 66: 96A.

10 Weir RE, Brennan PJ, Butlin CR, Dockrell HM. Use of a whole blood assay to evaluate in vitro T-cell responses to new leprosy skin test antigens in leprosy patients and healthy subjects. Clin Exp Immunol, 1999; 116: 263-269.

11 Streeton JA. Desem N. Jones SL. Sensitivity and specificity of a gamma interferon blood test for tuberculosis infection. Int J Tuberc Lung Dis, 1998; 2: 443-450.

12 Janeway CA. Travers P. Immunobiology: the immune system in health and disease, 2nd edn. Churchill Livingstone, Edinburgh, 1996; 9:40-9:42.

13 Chu CQ, Field M, Andrew E, Haskard D, Feldmann M, Maini RN. Detection of cytokines at the site of tuberculininduced delayed-type hypersensitivity in man. Clin Exp Immunol, 1992; 90: 522-529.

14 Manandhar R, LeMaster J, Roche P. Interferon gamma responses to candidate leprosy skin test reagents detects exposure to leprosy in an endemic population. Int J Lepr, 1999 (in press).

15 Higgins JA, Lazar CL, Brennan PJ, TerLouw SM. Two new leprosy skin test antigens: MLSA-LAM and MLCwA. Final report of phase I testing. BB-IND 7938, Report to US Federal Drug Administration, 1999.

16 Dockrell HA, Hussain R, Shahid F, Chiang T, Roche P, Butlin R, Pessolani C, Sampaio E, Sarno E, Gebre N, Hunegnaw M, Negesse Y, Amare G, Britton S, Murrillo L, Guzman F, Robertson B, Thole J, Young D, Fruth U. WHO synthetic peptide skin test antigens. Transactions of the 15 th International Leprosy Congress. Int J Lepr, 1998; 66: 95A.

Mycobacterial Research Laboratory

J. W. LeMAster \& P. Roche

Anandaban Leprosy Hospital

PO Box 151

Kathmandu, Nepal 